\title{
Stevens's law for time: A direct comparison of prospective and retrospective judgments
}

\author{
Simon Grondin ${ }^{1}$ • Vincent Laflamme ${ }^{1}$
}

Published online: 23 April 2015

(C) The Psychonomic Society, Inc. 2015

\begin{abstract}
Participants are aware that they have to perform a temporal task in a prospective timing condition but not in a retrospective timing condition. In the present study, a direct comparison of temporal estimates under each paradigm is proposed via a strategy where each participant is restricted to only 1 response. Participants were assigned to either a prospective or retrospective testing condition and asked to reproduce and then estimate verbally 1 of 6 intervals lasting .5 to $16 \mathrm{~s}$. The analyses based on Stevens's power law were restricted to intervals lasting 2 to $16 \mathrm{~s}$. With a verbal estimate method, the results indicate that the exponent is higher in retrospective than in prospective conditions (1.20 vs. 1.10 for females and 1.31 vs. 1.02 for males, respectively). For the interval reproduction task, the exponent based on Eisler's (1975) model was slightly higher for males (1.13) than for females (1.08) in prospective timing, but slightly higher for females (1.10) than for males (1.04) in retrospective timing. The results based on inferential statistics and the 6 intervals reveal that, with the verbal estimate method, females make significantly larger relative verbal estimates than males and, at $16 \mathrm{~s}$, intervals were judged as longer in the retrospective than in the prospective condition; with the reproduction method, the perceived duration is about the same in each paradigm and there is no significant sex effect. Overall, the data do not confirm that temporal intervals are perceived as longer in the prospective than in the retrospective conditions.
\end{abstract}

Keywords Prospective and retrospective timing - Sex effect . Stevens's law $\cdot$ Temporal processing

Simon Grondin

simon.grondin@psy.ulaval.ca

1 École de Psychologie, Université Laval, Québec, Québec, Canada G1V 0A6
One critical distinction for understanding some research findings in the field of time perception is related to the fact that a participant in an experiment might or might not know that the duration of a sensory signal, or of a given activity, has to be timed (Bisson \& Grondin, 2013; Block, 1992; Block \& Zakay, 1997; Brown, 1985). When a participant knows that a time interval will have to be estimated - what is called a prospective timing condition - paying attention to time during the sensory signal or the activity becomes relevant. When a participant is unaware that time is a concern-a retrospective timing condition - the estimation of the duration of this signal or activity will likely require some reconstruction in memory.

There is a general report about the relative duration of time intervals judged prospectively versus retrospectively: Intervals judged prospectively are most often reported to be perceived as longer than intervals judged retrospectively (Block \& Zakay, 1997; for a review, see Tobin, Bisson, \& Grondin, 2010). Moreover, retrospective judgments are reported to be much more variable than prospective ones (Block \& Zakay, 1997).

Beyond the fact of being aware or not that a temporal estimation will be required, the comparison of these paradigms suffers from two fundamental methodological differences. On the one hand, the interval length under investigation is usually briefer in the prospective than in the retrospective paradigm. It is easy to understand that studying the perception of long intervals in prospective conditions exposes experimenters to the possibility that a participant decides to use some internal strategies (like the explicit counting of chronometric units) for segmenting them into a series smaller subintervals. Such strategies, which are proved to be efficient (Grondin \& Killeen, 2009; Grondin, Meilleur-Wells, \& Lachance, 1999), are not possible in retrospective conditions. On the other hand, retrospective and prospective timing data most often differ on the basis of the number of trials completed in a given experimental condition. The study of prospective 
timing most often involves multiple estimations of intervals, and the variability of judgments is as important in assessing performances as the mean estimates are. With retrospective timing, it is not possible to adopt such an approach. After completing one trial, the participant is informed that time is a concern. At best, it is possible to first have the participant involved in a series of activities and then ask retrospectively to estimate the duration of each of these activities (Grondin \& Plourde, 2007).

Prospective and retrospective time judgments are argued to be based on the output of different cognitive mechanisms. Classical interpretations of the results in retrospective timing conditions refer to the notion of space occupied in memory by a certain number of activities occurring during the interval to be estimated (Ornstein, 1969), or the number of changes occurring during this interval (Block, 1992; Block \& Zakay, 2008). As for prospective timing, it is known to be under control of attentional mechanisms (Brown, 2008). The preparation of attention before the interval to be judged (Grondin \& Rammsayer, 2003; Los \& Horoufchin, 2011), and the disruption of attention by interferences from different sources during the intervals (Grondin, 2001) will alter perceived duration. The most critical and replicated finding about the role of attention on time perception stems from studies involving a dual-task strategy (Brown, 2008), where a participant is required to perform simultaneously a temporal and a nontemporal task (Brown, 1985), sometimes with a restriction regarding the amount of attention allocated to each task (Macar, Grondin, \& Casini, 1994). In general, with more attention allocated to time, duration is perceived as longer.

Only a few studies compared prospective and retrospective timing directly, and, in most cases, the comparison is based actually on the effect of a dual-task condition (see, for instance, Hicks, Miller, \& Kinsbourne, 1976; and for a review, see Block \& Zakay, 1997). In these studies, the objective is to see if these two types of timing paradigms share some common properties. Most results indicate that a reduction of attention has more impact on perceived duration in the prospective than in the retrospective condition. Nevertheless, some authors argue that a common timing process may underlie prospective and retrospective timing (Brown \& Stubbs, 1988). For instance, in the study by Brown (1985), the impact of an additional nontemporal task was the same in both the prospective and the retrospective conditions. In another type of study, Boltz (2005) showed that with the repetitive exposure to naturalistic coherent events (i.e., temporal periodicity between successive body movements), retrospective judgments become as accurate as prospective judgments. This effect holds with visual or auditory events and does not occur when incoherent events are used.

What is making this prospective versus retrospective conditions comparison possible in a study like the one reported by
Brown (1985) is (1) the fact that only one judgment was required in the prospective condition, contrary to most psychophysical studies involving prospective judgments about time; and (2) that a between-subjects design was used. In the present study, we opted for such a method. This allowed a direct comparison of the results obtained with the prospective and retrospective paradigms. Each participant was asked to complete a single trial and was thus subjected to a single experimental condition.

In the present study, no comparison was based on the effect of a dual-task condition. The novelty of the approach lies in the intention to make a direct comparison for many target durations (e.g., six targets, from .5 to $16 \mathrm{~s}$ ). Indeed, it is intended to draw a psychophysical function in the retrospective and in the prospective conditions. In the context of time, a psychophysical function captures the general growth of psychological time as a function of chronometric time. Such a psychophysical approach is usually restricted to prospective timing, considering that multiple trials are required from each participant for drawing the function.

Another feature of the present study is novel. Instead of asking participants to go in a laboratory for a very brief experimentation, the experiment took place at several public locations. The purpose of this strategy was not only to increase the sample size but also to reduce the possibility that participants of the retrospective timing group were aware of the requirement to estimate time when entering in a laboratory specialized in research on time perception. Finally, with only one trial and, thus, without knowing the length of the interval to be timed, it is not possible for a participant to determine if it is worth adopting a counting strategy.

\section{Method}

\section{Participants}

A total of 817 persons (410 females, 407 males) volunteered for participating in this experiment. They were recruited in public places (on the campus of Université Laval and in neighboring shopping centers) and participated immediately after completing a consent form. Their ages ranged from 18 to 83 years old $(M=29.13, M d n=23)$. The participation lasted no more than 5 minutes.

More specifically, although each participant was invited to complete both tasks (reproduction and verbal estimate), not all data were kept for analyses, as described below. Nevertheless, for the reproduction task, there were from 23 to 34 participants (lowest and highest mean ages from 24.3 to 33.7 ) in each of the 12 conditions ( 6 durations $\times 2$ sex) in the prospective condition, and from 23 to 36 participants (lowest and highest mean ages from 24.0 to 32.5 ) in the retrospective condition. For the verbal estimate task, there were from 21 to 32 
participants (lowest and highest mean ages from 24.1 to 34.0) in each of the 12 conditions in the prospective condition, and from 21 to 34 participants (lowest and highest mean ages from 24.4 to 35.4 ) in the retrospective condition.

\section{Apparatus and stimuli}

Participants were seated in front of a portable computer (ThinkPad) running the experiment, and they used headphones (Sennheiser HD 477) connected to this computer. Two types of stimuli were used, one visual (a photo of mountains and the sea) and one auditory ( $1 \mathrm{kHz}$, about $70 \mathrm{~dB}$ SPL).

\section{Procedure}

This study included only a single trial made of three steps. The first step was the stimuli presentation. The 20 -ms auditory signal was presented, followed immediately by the presentation of the visual stimulus on the computer screen. The photo was presented for the target duration $(.5,1,2,4,8$, or $16 \mathrm{~s})$, followed by the presentation of the brief auditory signal again. The .5 -s value looks like a critical one for this type of psychophysical investigation, at least with magnitude estimation (Eisler, 1976). The 16-s value is the briefer of two standard durations used by Brown (1985), who compared directly prospective and retrospective conditions.

The second step was for time reproduction. Participants were instructed to reproduce the duration between the two auditory signals by pressing twice on the space bar of the keyboard (once to start the reproduction and again when the same duration had elapsed). The last step was verbal estimation. The participants were asked to estimate, to the nearest 10th of a second, the duration between the two auditory signals as well as the minimum and maximum possible durations of this interval. Participants were asked to reproduce the interval and then, to estimate duration verbally, in this specific order, for avoiding, with longer intervals, the adoption of a counting strategy (of seconds) during the reproduction, after having estimated time in chronometric units. After the verbal estimation, participants also had to answer to two questions: (1) "Were you aware that you would have to reproduce and estimate time before we presented the picture?" and (2) "Did you look at a watch or a clock during the experiment?" Then, they signed a post facto consent form, explaining the real purpose of this experiment.

There were two main experimental conditions in this experiment. Half of the participants were in the prospective condition and half were in the retrospective condition. In the prospective condition, participants were told in advance that they would have to reproduce and estimate duration. In the retrospective condition, participants were told about the presentation of the three stimuli (a visual stimulus between two brief auditory signals). They were told that the photo of mountains and the sea would be presented for an undetermined amount of time and that they should pay attention to this photo because they would eventually be asked questions about it after the presentation.

\section{Data analyses}

An initial look at the data indicated that many participants did not understand the instructions for the tasks they were asked to complete. In a first step of the data filtration process, we removed the data from participants who looked at a watch or a clock during the experiment, or who did not adhere to the prospective or retrospective design. That is, we removed the participants in the retrospective condition who reported that they were aware of the true nature of the task while the target interval was presented, and we removed the participants in the prospective design that were not aware of that fact. Eightyseven participants were removed (48 in the prospective paradigm and 39 in the retrospective). Of the remaining 730 participants, 17 completed only the temporal reproduction task. For this reason, the rest of the filtration process was applied separately for each task. For the next step of the filtration process, we removed the outliers. They were determined separately for each cell of the experimental design. In order to be considered an outlier, the logarithm of the reproduced or estimated duration of a participant had to be either 2.5 interquartile range below the first quartile or 2.5 interquartile range above the third quartile. For the reproduction task, the data from 55 participants were rejected (final $N=675$ ), and for the verbal estimation task, 27 outliers were removed (final $N=$ 686).

\section{Results}

The mean data in each experimental condition for male and for female are presented in Figs. 1 (Reproduced interval/target) and 2 (verbal estimate/target). Before presenting inferential statistics for comparing experimental conditions, the data are analyzed in the light of Stevens's power law.

\section{Stevens's power law}

The relationship between subjective time $(S)$ and chronometric time $(\phi)$ is quantified with the estimate the exponent, $b$, of Stevens's power law:

$S=K \phi^{b}$.

It is this equation that is used for analyzing data obtained with verbal estimates. A $b$ value lower than 1 indicates that 
Fig. 1 Mean relative reproduced interval in the prospective and retrospective judgment conditions as a function of the target duration for females (upper panel) and males (lower panel). Bars are standard errors

\section{Females}

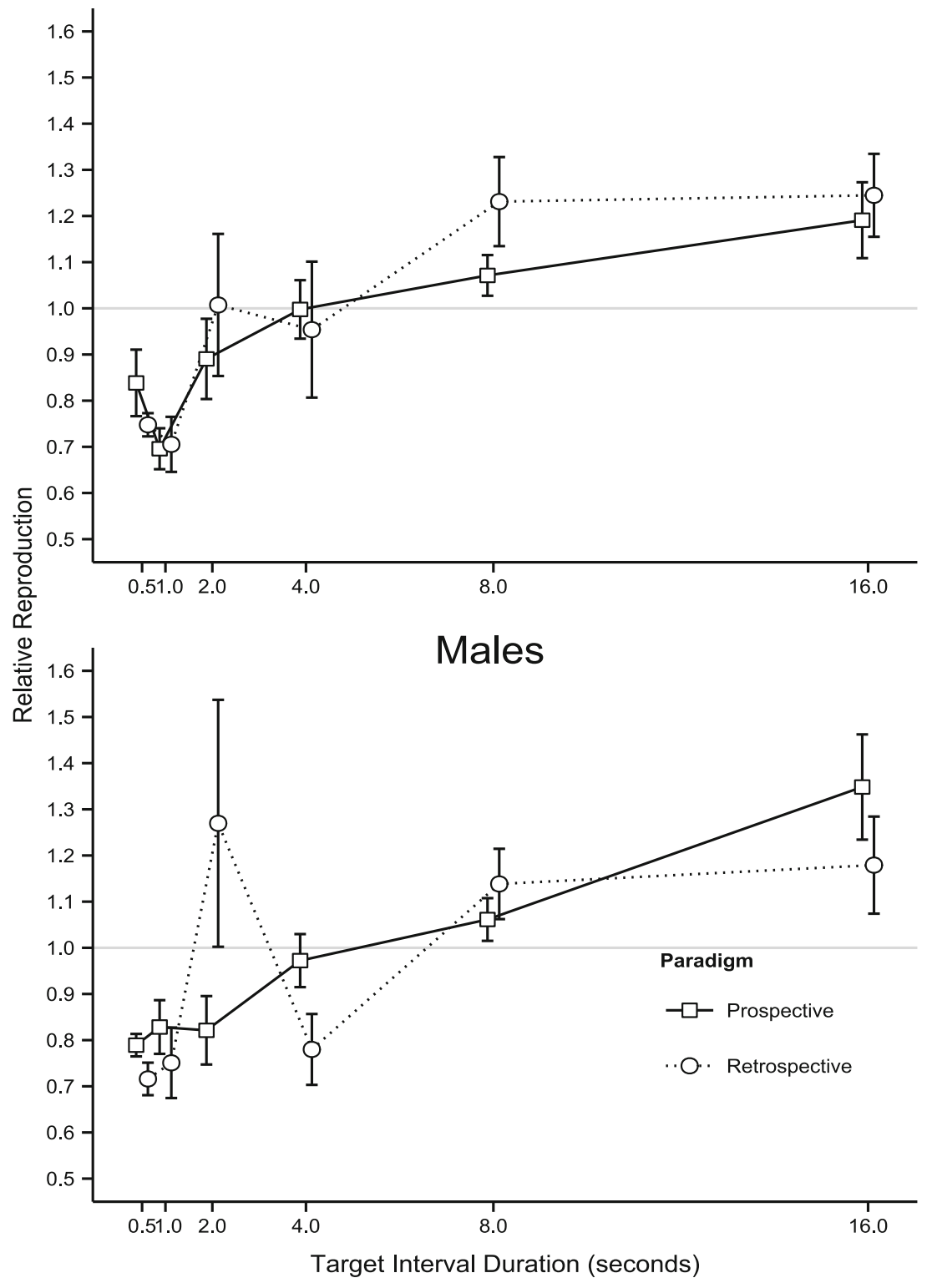

subjective time tends to grow slower as duration becomes longer, and a $b$ value higher than 1 indicates that subjective time rather tends to grow faster as duration becomes longer.

For calculating the $b$ exponent obtained with the temporal reproduction method, we rather used an approach based on Eisler's (1976) analysis. Assuming Eisler's (1975) parallel-clock model, these values are calculated from the slope of the linear regression between the magnitudes of the reproduction and the total duration (target + reproduction). Since the dependent variable of this regression (the reproduced duration) shares all of its variance with the independent variable (total duration), the analyses were run on the geometric mean of the reproduced durations. According to Equation 3a in
Eisler (1976), if $a$ is the estimated slope of the linear regression between reproduced and total duration, then:

$b=\frac{\log (.5)}{\log (a)}$.

For both reproduction data and verbal estimates, the regression analyses are restricted to intervals lasting 2 to $16 \mathrm{~s}$ because data at .5 and $1 \mathrm{~s}$ are less reliable. Contrary to Eisler (1976), no subjective zero was taken into account, so the regressions were run using no intercept.

For the reproduction data, the estimates of $b$ based on Eisler's method (slope and goodness of fit in parentheses) are $1.134\left(a=.542, R^{2}=.998\right)$ for males in the prospective 
condition and $1.071\left(a=.523, R^{2}=.999\right)$ for females, and $1.041\left(a=.514, R^{2}=.999\right)$ for males in the retrospective condition and $1.103\left(a=.533, R^{2}=.999\right)$ for females.

For the verbal estimation, the value of parameters $K$ and $b$ (Eq. 1) was estimated with linear regression and revealed by the intercept and slope of the function with log-log coordinates. The estimates are $b=1.015, K=1.168\left(R^{2}=.738\right)$ for the males' prospective timing and $b=1.103, K=1.107\left(R^{2}=\right.$ $.645)$ for the females; and $b=1.310, K=0.684\left(R^{2}=.724\right)$ for males retrospective timing and $b=1.196, K=1.027\left(R^{2}=\right.$ .644) for females. With both females and males, the exponent value is higher in retrospective than in prospective condition.

\section{Inferential statistics}

There are three independent variables: the prospective versus retrospective paradigm, duration (.5 to $16 \mathrm{~s})$, and the sex of participants.

\section{Reproduced intervals}

As indicated earlier (see Fig. 1), and in order to make the direct comparison of duration conditions possible, the dependent variable analyzed is the relative reproduction: reproduced interval/target. Note first that the relative reproductions are weakly but significantly correlated with the age of the participants, and that is true for prospective timing, $r=.275, p<.01$, and retrospective timing, $r=.211, p<.01$.

A completely randomized factorial design ( 2 Paradigm $\times 2$ Sex $\times 6$ Duration) ANOVA on the relative reproductions revealed no main effect of paradigm $(p=.66)$ and $\operatorname{sex}(p=.87)$, but a significant duration effect, $F(5,651)=14.55, p<.01, \eta_{p}^{2}$ $=.10$. There was a statistically significant duration $\times$ paradigm interaction effect, $F(5,651)=2.35, p=.04, \eta_{p}^{2}=.018$. None of the other interaction effects are significant $(p=.43$, for the triple interaction effect).

\section{Verbal estimates}

As for the other task, the dependent variable analyzed is the relative verbal estimate: verbal estimate/target. Except for the mean results of males in the retrospective condition at $4 \mathrm{~s}$, all durations were overestimated (see Fig. 2). The relative verbal estimates were not correlated with the age of the participants, neither in the prospective, $r=.084, p=.12$, nor in the retrospective condition, $r=.087, p=.10$.

The same ANOVA design as the one described above was used to analyze data. It reveals that both the sex, $F(1,662)=$ $10.38, p<.001, \eta_{p}^{2}=.015$, and the duration effect, $F(5,662)=$ $4.83, p<.001, \eta_{p}^{2}=.035$, are significant, and the paradigm effect is not significant, $F(1,662)=0.74, p=.39, \eta_{p}^{2}=.001$. The only significant interaction involves duration and paradigm, $F(5,662)=2.65, p=.022, \eta_{p}^{2}=.02$. The data indicate some overestimation of the 4-s interval in the prospective condition compared to the retrospective one, $F(1,105)=$ $3.13, p=.08, \eta_{p}^{2}=.029$, and the reversed pattern at $16 \mathrm{~s}$, $F(1,109)=11.94, p<.001, \eta_{p}^{2}=.099$.

When the target durations of 0.5 and $1 \mathrm{~s}$ are excluded from the analysis, the pattern of results is essentially the same. There is still a statistically significant main effect of sex, $F(1,440)=8.39, p=.004, \eta_{p}^{2}=.019$, and of target duration, $F(3,440)=4.15, p=.0064, \eta_{p}^{2}=.028$, and the paradigm effect is still not statistically significant, $F(1,440)=2.40, p$ $=.12, \eta_{p}^{2}=.0054$. Again, the only significant interaction effect involves duration and paradigm, $F(3,440)=3.74, p=.011$, $\eta_{p}^{2}=.025$.

\section{Discussion}

There are essentially three main findings in the present investigation. First and most importantly, from a psychophysical law standpoint, the value of the exponent $b$ is slightly higher in the retrospective than in the prospective condition with verbal estimates, but such a difference with the reproduction task depends on sex. Second, there is a sex effect when the verbal estimate method is used. Finally, with verbal estimates, there is a paradigm effect, but this effect interacts with the duration range factor.

\section{Stevens's power law}

The present investigation reveals that the value of the exponent of Stevens's power law, $b$, for retrospective and prospective timing varies as a function of the method employed. It is important to remind that the analyses were restricted to the 2 to $16 \mathrm{~s}$ portion of the data. The value for male and female is slightly higher in retrospective condition than in prospective condition with the verbal estimates. For the interval reproduction task, which is based on a different analysis method (Eisler, 1975, 1976), it is the sex difference that is striking: this difference occurred in prospective timing, but not in retrospective timing.

With the reproduction method, the exponents were higher than 1 . The $b$ value was about 1.13 for male and 1.07 for female in the prospective timing condition. Strictly speaking, this implies that the flow of time is faster for longer than for briefer intervals. Such an interpretation is also valid with the verbal estimation method where the $b$ values were about the same: 1.02 for male and 1.10 for female. In both cases, the values are higher than the mean exponent, .9 , retained by Eisler (1976) at the term of his extensive review on the question (studies involving prospective timing). Allan (1979) rather argued that 1 better reflects the form of the psychophysical law in time perception. Bobko, Thompson, and Schiffman (1977) noted that the psychophysical method (verbal or 
Fig. 2 Mean relative verbal estimates in the prospective and retrospective judgment conditions as a function of the target duration for females (upper panel) and males (lower panel). Bars are standard errors

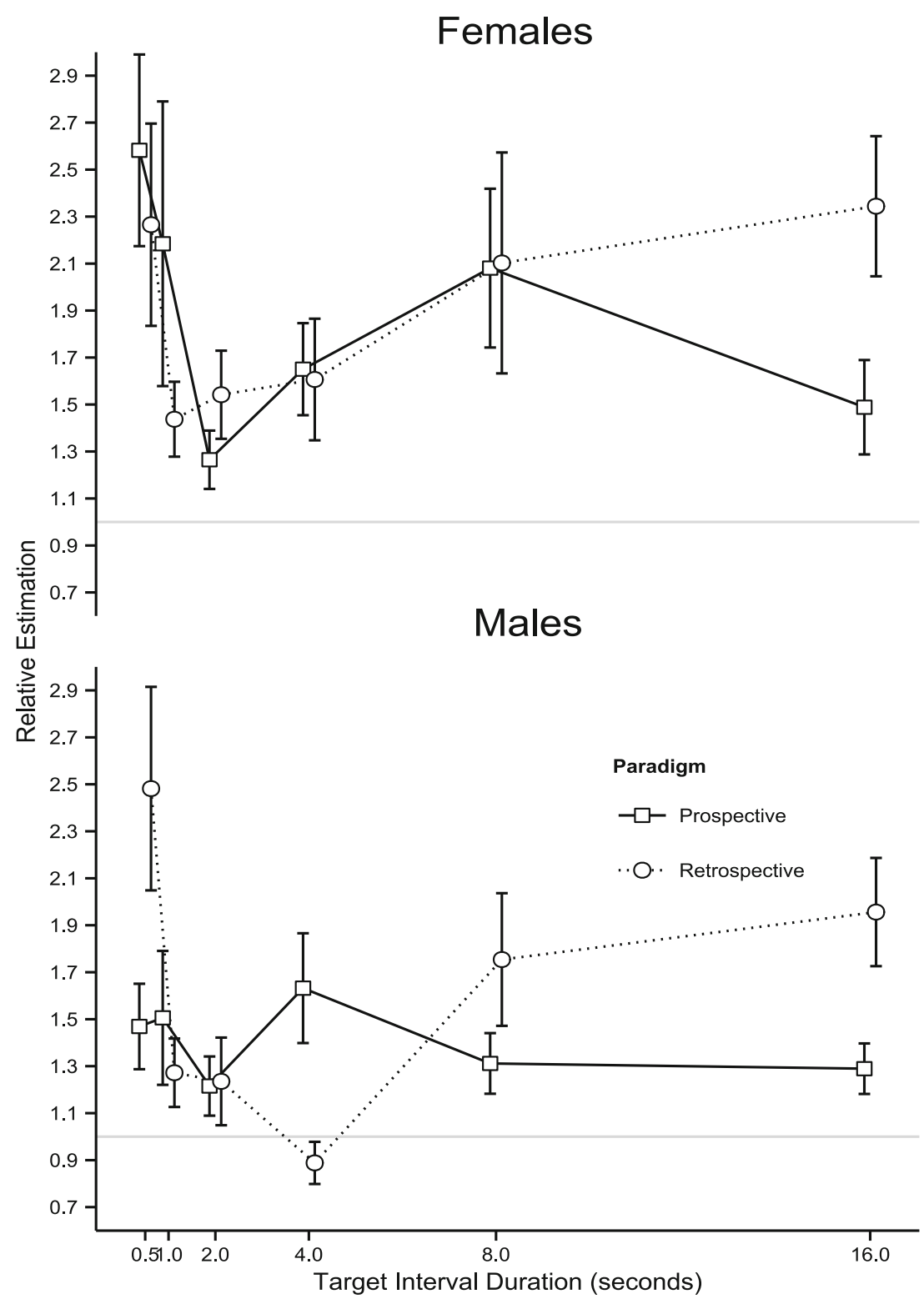

magnitude estimation, with or without modulus) does not exert much influence on the exponent value (between .90 and .99 for intervals from $250 \mathrm{~ms}$ to $5 \mathrm{~s}$ ). The exponents in the present study are somewhat surprising, given the mean values proposed by Eisler (1976) or Allan (1979) as a general picture for time perception. However, the difference is not that large if we compare for instance with exponent values of 2.13 or .40, as is observed for electric shock and loudness, respectively (Stevens, 1975).

With the verbal estimates, the flow of time is faster for longer than for briefer intervals in the retrospective condition ( $b>1.2$ for both sex), a verdict that is not that clear with prospective timing ( $b$ being only slightly higher than 1). This could be interpreted as if paying attention (prospective) or not (retrospective) to time has an incidence on the flow of time, with attention, $b$ remains closer to 1 . However, the fact that, with the reproduction method, male participants have a higher $b$ value in prospective than in retrospective timing reveals that whether or not someone pays attention to time during a given period to be timed is probably not the critical factor determining the exponent value of the power law.

Of course, the present findings should be taken with circumspection. Ideally, in psychophysics, a power law would be analyzed on the basis of individual functions, with multiple estimates per data points. This was obviously not possible in the present study since the main goal was to contrast retrospective and prospective timing. The data remain theoretically significant 
considering that the same method was applied to each paradigm and that there were many participants for estimating the mean value in each condition.

\section{Sex, paradigm, and duration}

As for the effect of sex on subjective time, the present investigation leads to results requiring nuances. The data collected with the reproduction method show no indication of a sex effect. However, with verbal estimates, there is a main effect of sex. Overall, females $(M=1.86)$ tend to make larger relative verbal estimates than males $(M=1.53)$. This difference holds even when considering only target durations longer than 1 second (females' $M=1.74$ and males' $M=1.43$ ). These results are consistent with the output of a meta-analysis reported by Block, Hancock, and Zakay (2000). They noted females exhibit a larger ratio of subjective-to-objective duration than males do in retrospective conditions. Their conclusion for prospective timing requires more nuances: females give larger verbal estimates than males, but there is no significant difference with the reproduction method. In the present study, sex difference would not depend on the prospective versus retrospective condition of the experiment. It would however depend on the method (reproduction vs. verbal estimate) used for investigating psychological time. It would be more in the way of using chronometric units that males and females differ.

With the verbal estimate method, a paradigm by duration interaction was significant, and the pattern explaining this interaction is the same for males and females. What is interesting is to contrast the data obtained at $16 \mathrm{~s}$ with verbal estimates with those obtained in the reproduction task. While the relative reproduction tended to slightly increase from 8 to $16 \mathrm{~s}$ in prospective condition and to remain stable in retrospective condition, the relative verbal estimates increased a lot from 8 to $16 \mathrm{~s}$ in the retrospective condition and rather tended to decrease in the prospective condition. Note that this finding is consistent with the large overestimation of 15-min intervals judged retrospectively (Grondin, Laflamme, Bisson, \& Désautels, 2014).

Finally, it should be noted that the reproduction task was completed first, before the verbal estimate. Though speculative, it is not possible to discard the hypothesis that what was taken into account during the verbal estimates is probably not restricted to the original (presented) interval; the reproduced interval may interfere somehow. The difference between the reproduced and verbally estimated intervals at $16 \mathrm{~s}$ is a bit surprising considering the results reported by Brown (1985) in his control conditions. At $16 \mathrm{~s}$, the ratio scores reported in the verbal estimate and reproduction conditions, respectively, were .93 and .92 in the prospective condition and .80 and .78 in the retrospective condition (Experiment 1); and, in the same order (Experiment 2), .90 and .98 and .54 and .89. Note that in
Brown's experiments, verbal estimates were made before the reproduction of intervals.

\section{Conclusion}

The study shows that the exponent's value of Stevens's power law is not exactly the same for prospective and retrospective timing when verbal estimates are used, but the data obtained with the reproduction method indicate that the difference between the types of timing depends on the sex of participants. The part of the study based on inferential statistics rather reveals a significant sex effect with verbal estimates, but not with the reproduction task. Finally, at $16 \mathrm{~s}$, intervals were judged as longer in the retrospective than in the prospective condition with verbal estimates, but not with the reproduction task.

Authors note This research was made possible by a research grant awarded by the Natural Sciences and Engineering Council of Canada to SG. We would like to express gratitude to Simon Tobin and Nicolas Bisson for discussions about this project and to Åke Hellström for his numerous and generous suggestions about data analyses. We would like to extend special thanks to Pierre-Yves Bergeron, Philippe Bienvenue, Marie-Pier Brien, Dominique Caron-Bélanger, Félix Désautels, Daniel Fortin-Guichard, Jason Vaillancourt, Katherine Labonté, and Jason Vaillancourt for their help with data collection. Correspondence should be addressed to Simon Grondin, École de psychologie, 2325 rue des Bibliothèques, Université Laval, Québec, Qué., Canada G1A 0 V6. Email: simon.grondin@psy.ulaval.ca

\section{References}

Allan, L. G. (1979). The perception of time. Perception \& Psychophysics, 26, 340-354.

Bisson, N., \& Grondin, S. (2013). Time estimates of Internet surfing and video gaming. Timing \& Time Perception, 1, 39-64.

Block, R. A. (1992). Prospective and retrospective duration judgment: The role of information processing and memory. In F. Macar, V. Pouthas, \& W. J. Friedman (Eds.), Time, action and cognition: Towards bridging the gap (pp. 141-152). Dordrecht, Germany: Kluwer Academic.

Block, R. A., Hancock, P. A., \& Zakay, D. (2000). Sex differences in duration judgments: A meta-analytic review. Memory \& Cognition, $28,1333-1346$.

Block, R. A., \& Zakay, D. (1997). Prospective and retrospective duration judgments: A meta-analytic review. Psychonomic Bulletin \& Review, 4, 184-197.

Block, R. A., \& Zakay, D. (2008). Timing and remembering the past, the present, and the future. In S. Grondin (Ed.), Psychology of time (pp. 367-394). Bingley, UK: Emerald Group.

Bobko, D. J., Thompson, J. G., \& Schiffman, H. R. (1977). The perception of brief temporal intervals: Power functions for auditory and visual stimulus intervals. Perception, 6, 703-709.

Boltz, M. G. (2005). Duration judgments of naturalistic events in the auditory and visual modalities. Perception \& Psychophysics, 67, $1362-1375$. 
Brown, S. W. (1985). Time perception and attention: The effects of prospective versus retrospective paradigms and task demands on perceived duration. Perception \& Psychophysics, 38, 115-124.

Brown, S. W. (2008). Time and attention: Review of the literature. In S. Grondin (Ed.), Psychology of time (pp. 111-138). Bingley, UK: Emerald Group.

Brown, S. W., \& Stubbs, D. A. (1988). The psychophysics of retrospective and prospective duration timing. Perception, 17, 297-310.

Eisler, H. (1975). Subjective duration and psychophysics. Psychological Review, 82, 429-450.

Eisler, H. (1976). Experiments on subjective duration 1878-1975: A collection of power function exponents. Psychological Bulletin, $83,185-200$.

Grondin, S. (2001). Time psychophysics and attention. Psychologica, 28, 177-191.

Grondin, S., \& Killeen, P. R. (2009). Tracking time with song and count: Different Weber functions for musicians and non-musicians. Attention, Perception, \& Psychophysics, 71, 1649-1654.

Grondin, S., Laflamme, V., Bisson, N., \& Désautels, F. (2014). The delay before recall changes the remembered duration of 15 -min video sequences. Applied Cognition Psychology, 28, 677-684. doi:10. 1002/acp.3053

Grondin, S., Meilleur-Wells, G., \& Lachance, R. (1999). When to start explicit counting in a time-intervals discrimination task: A critical point in the timing process of humans. Journal of Experimental Psychology: Human Perception and Performance, 25, 993-1004.

Grondin, S., \& Plourde, M. (2007). Judging multi-minute intervals retrospectively. Quarterly Journal of Experimental Psychology, 60, 1303-1312.

Grondin, S., \& Rammsayer, T. (2003). Variable foreperiods and temporal discrimination. The Quarterly Journal of Experimental Psychology A: Human Experimental Psychology, 56A, 731-765.

Hicks, R. E., Miller, G. W., \& Kinsbourne, M. (1976). Prospective and retrospective judgements of time as a function of amount of information processed. American Journal of Psychology, 89, 719-730.

Los, S. A., \& Horoufchin, H. (2011). Dissociative patterns of foreperiod effects in temporal discrimination and reaction time tasks. Quarterly Journal of Experimental Psychology, 64, 1009-1020. doi:10.1080/ 17470218.2010.532225

Macar, F., Grondin, S., \& Casini, L. (1994). Controlled attention sharing influences time estimation. Memory \& Cognition, 22, 673-686.

Ornstein, R. (1969). On the experience of time. New York, NY: Penguin.

Stevens, S. S. (1975). Psychophysics: Introduction to its perceptual, neural and social prospects. New York, NY: Wiley.

Tobin, S., Bisson, N., \& Grondin, S. (2010). An ecological approach to prospective and retrospective timing of long durations: A study involving gamers. PLoS ONE, 5(2), e9271. 